\title{
Enhanced transmission and beaming via a zero-index photonic crystal
}

Hodjat Hajian, Ekmel Ozbay, and Humeyra Caglayan

Citation: Appl. Phys. Lett. 109, 031105 (2016); doi: 10.1063/1.4959085

View online: https://doi.org/10.1063/1.4959085

View Table of Contents: http://aip.scitation.org/toc/apl/109/3

Published by the American Institute of Physics

\section{Articles you may be interested in}

Long-range Tamm surface plasmons supported by graphene-dielectric metamaterials

Journal of Applied Physics 121, 033101 (2017); 10.1063/1.4973900

Unidirectional transmission using array of zero-refractive-index metamaterials

Applied Physics Letters 104, 193509 (2014); 10.1063/1.4878400

Acoustic metasurface-based perfect absorber with deep subwavelength thickness

Applied Physics Letters 108, 063502 (2016); 10.1063/1.4941338

Photon management with index-near-zero materials

Applied Physics Letters 109, 051101 (2016); 10.1063/1.4960150

Design of zero index metamaterials with PT symmetry using epsilon-near-zero media with defects Journal of Applied Physics 121, 094503 (2017); 10.1063/1.4977692

Super-reflection and cloaking based on zero index metamaterial

Applied Physics Letters 96, 101109 (2010); 10.1063/1.3359428

\section{PHYSICS TODAY}

MANAGER'S GUIDE

WHITEPAPERS

\section{READ NOW}

PRESENTED BY

Accelerate R\&D with
Multiphysics Simulation 


\title{
Enhanced transmission and beaming via a zero-index photonic crystal
}

\author{
Hodjat Hajian, ${ }^{1, a)}$ Ekmel Ozbay, ${ }^{1,2,3}$ and Humeyra Caglayan ${ }^{1,4}$ \\ ${ }^{1}$ Nanotechnology Research Center, Bilkent University, 06800 Ankara, Turkey \\ ${ }^{2}$ Department of Physics, Bilkent University, 06800 Ankara, Turkey \\ ${ }^{3}$ Department of Electrical and Electronics Engineering, Bilkent University, 06800 Ankara, Turkey \\ ${ }^{4}$ Department of Electrical and Electronics Engineering, Abdullah Gul University, 38080 Kayseri, Turkey
}

(Received 3 June 2016; accepted 7 July 2016; published online 19 July 2016)

Certain types of photonic crystals with Dirac cones at the $\Gamma$ point of their band structure have a zero effective index of refraction at Dirac cone frequency. Here, by an appropriate design of the photonic structure, we obtain a strong coupling between modes around the Dirac cone frequency of an alldielectric zero-index photonic crystal and the guided ones supported by a photonic crystal waveguide. Consequently, we experimentally demonstrate that the presence of the zero-index photonic crystal at the inner side of the photonic crystal waveguide leads to an enhancement in the transmission of some of the guided waves passing through this hybrid system. Moreover, those electromagnetic waves extracted from the structure with enhanced transmission exhibit high directional beaming due to the presence of the zero-index photonic crystal at the outer side of the photonic crystal waveguide. Published by AIP Publishing. [http://dx.doi.org/10.1063/1.4959085]

Zero-index metamaterials that are matched to free space and have a near-zero refractive index can pass electromagnetic waves with the characteristic of a zero spatial phase change. ${ }^{1}$ This characteristic has led to significant attention being paid to these systems due to their outstanding capability in molding the propagation of light. Squeezing electromagnetic energy within very narrow channels, ${ }^{2,3}$ designing matched zero-index systems, ${ }^{4}$ shaping the radiation pattern of a source, ${ }^{5-7}$ super-reflection and cloaking, ${ }^{8,9}$ and enhancing light transmission through a subwavelength aperture ${ }^{10}$ can be referred to as the highlighted application examples of the zero-index metamaterials. These structures have been designed $^{11}$ and constructed ${ }^{12}$ with the use of chiral inclusions or sub-wavelength resonant metallic elements. Recently, in order to remove the significant Ohmic losses of these metal-based systems, the first all-dielectric zero-index metamaterial has been theoretically investigated and experimentally proved within the microwave range of frequency. ${ }^{13}$ In Ref. 13, it has been shown that some types of photonic crystals (PCs), with a high enough dielectric constant and appropriate rod radius can show Dirac cones at the $\Gamma$ point in their photonic band structure at a specific frequency. The Dirac cones of these PCs, which are a consequence of the accidental degeneracy not the lattice symmetry, are different from the ones exhibited at the $\mathrm{K}$ point in the band structure of graphene ${ }^{14}$ or other types of photonic crystals. ${ }^{15}$ Using effective medium theory, ${ }^{16}$ it has been proven that the effective permittivity $\left(\varepsilon_{e f f}\right)$ and permeability $\left(\mu_{e f f}\right)$ of the PCs with the Dirac cone at the $\Gamma$ point of their transverse magnetic (TM) band structure are simultaneously zero at the Dirac cone frequency. ${ }^{13}$ Consequently, their effective index of refraction, $n_{\text {eff }}$, is zero. These kinds of all-dielectric metamaterials thus can be considered as zero-index PCs (ZPCs), potentially capable of exhibiting extraordinary features like passing light with no change in the phase, and consequently lensing and cloaking. ${ }^{13}$ It should be noted that photonic

${ }^{a)}$ Electronic mail: hodjat.hajian@bilkent.edu.tr crystals are the structures with dielectric constant periodically varying in space, ${ }^{13}$ and they can be completely distinguished from metamaterials. However, once the above-mentioned conditions are satisfied, Mie resonances can be supported ${ }^{16}$ and, consequently, they induce intervals of negative effective permittivity and permeability of the system. ${ }^{18}$ In this situation, we should expect a transition from PCs to all dielectric metamaterials. ${ }^{19}$ Moreover, inspired by the ZPC proposed in Ref. 13, in an out-of-plane geometry, Moitra et al. ${ }^{20}$ experimentally demonstrated an all-dielectric zero-index metamaterial operating at optical frequencies that is potentially applicable to enhance the directivity of the spontaneous emission from quantum dot light sources embedded inside the structure. Moreover, Li et al. $^{21}$ fabricated an in-plane all-dielectric zero-index metamaterial which is fully compatible with on-chip integrated photonics, applicable in the telecom wavelength range.

On the other hand, it was theoretically shown ${ }^{22}$ and experimentally verified ${ }^{23}$ that by creating an appropriate surface corrugation and grating-like layer at the input and output sides of a PC waveguide (PC WG), light transmission through this system can be substantially enhanced and its extraction can be effectively modified leading to on- and offaxis beaming. ${ }^{24}$ In this Letter, we experimentally demonstrate that by adding a zero-index photonic crystal at the input side of a PC WG, light can be substantially funneled from free space into the waveguide at frequencies near the Dirac cone frequency of the ZPC. Furthermore, far-field radiation pattern proves that when a ZPC is added to the output side of the PC WG, it is possible to obtain electromagnetic waves extracted from the system within a narrow angular region.

The schematic of the considered system in this work is presented in Fig. 1(a). In order to investigate our main idea, we concentrate on two-dimensional PCs (2D PCs). The 2D PCs are composed of square arrays of the cylindrical rods of Alumina, $\varepsilon=9.61$, in air background. The PC waveguide is designed with lattice constant of $a_{P C}=9 \mathrm{~mm}$ and radius of 
$\Delta$

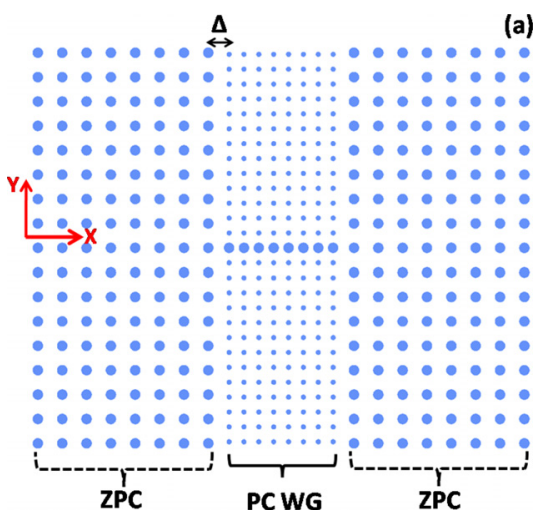

(a)
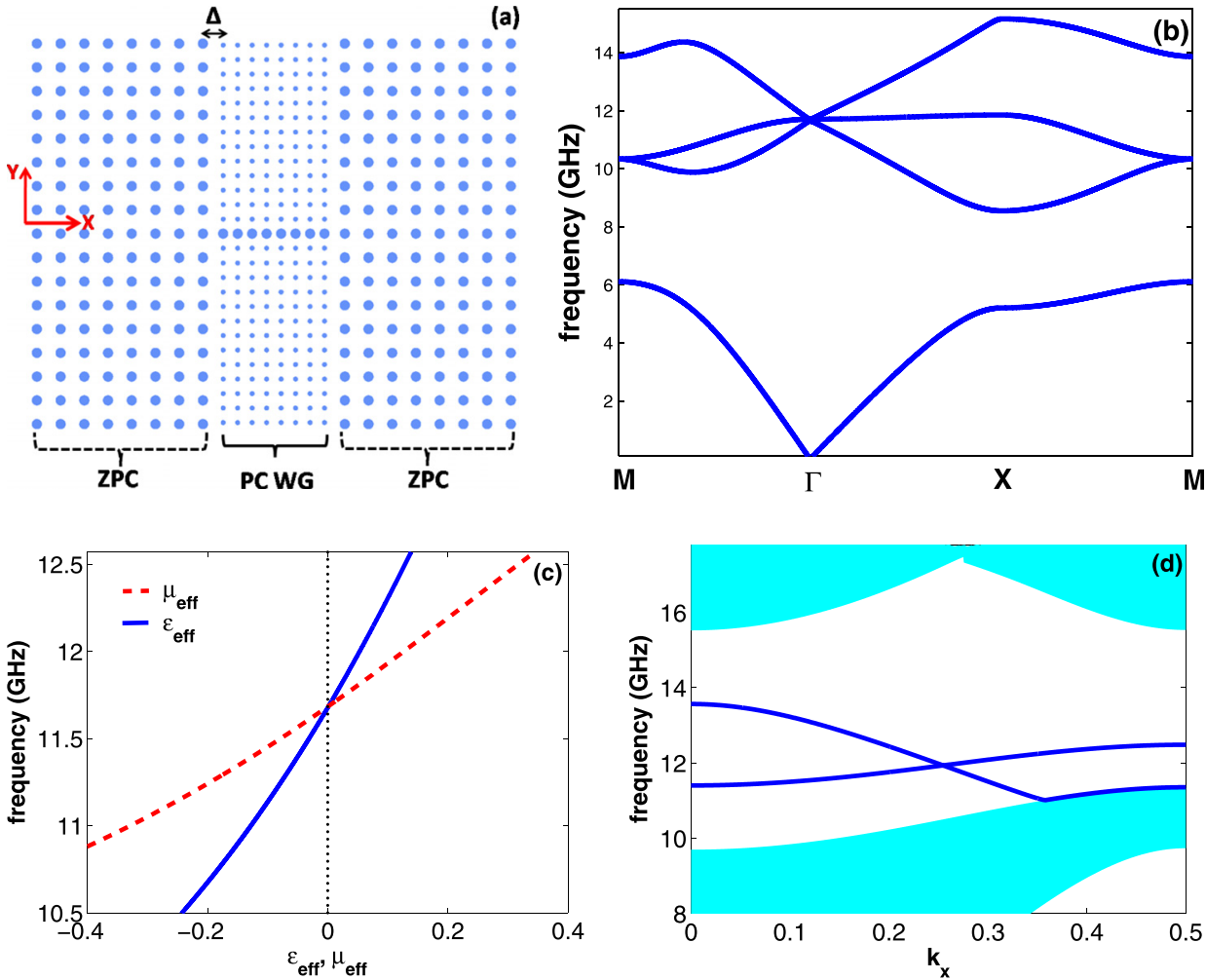

FIG. 1. Panel (a) shows the schematic of the hybrid system under our consideration for which ZPC is placed at both sides of the $\mathrm{PC}$ waveguide. The radius of the rods of the typical PC and ZPC are $r_{P C}$ and $r_{Z P C}$, and the air gap separating the PCs is $\Delta$. Panel (b) illustrates the band structure of the ZPC. Effective permittivity and permeability of the ZPC are shown in panel (c). At the Dirac cone frequency, $11.7 \mathrm{GHz}$ in panel (b), the effective parameters in panel (c) are zero. The projected band structure of the PC WG is represented in panel (d). In this plot, turquoise and white regions are allowed and forbidden bands of the PC, respectively. Solidblue curves in the bandgap are guided modes supported by the PC WG.
$r_{P C}=1.55 \mathrm{~mm}$ by replacing the central row of the PC with the rods of radius of $3.17 \mathrm{~mm}$. On the other hand, the lattice constant and the cylinders' radius for ZPC are $a_{Z P C}=14.75 \mathrm{~mm}$ and $r_{Z P C}=3.17 \mathrm{~mm}$, respectively. Moreover, the distance separating PC WG and ZPC, depicted by $\Delta$ in Fig. 1(a), is $1.4 a_{P C}$. Panel (b) of Fig. 1 illustrates TM band structure of the ZPC. As seen, around a specific frequency, this zeroindex PC shows linear dispersion and Dirac cones at the $\Gamma$ point in the band structure. The specific frequency, also referred to as the Dirac frequency or accidental degeneracy frequency, ${ }^{13}$ fits to $11.7 \mathrm{GHz}$ for the zero-index PC under our consideration. As comprehensively explained in Refs. 13 and 20, two branches with linear dispersions intersect with a flat band making a triply-degenerate point at $\mathbf{k}=0$. At this point, the flat band corresponds to a dipolar magnetic longitudinal mode (magnetic field parallel to $\mathbf{k}$ around $\Gamma$ point), while the Dirac cone modes match with dipolar magnetic transverse and electric monopolar modes. Fig. 1(c), which depicts $\varepsilon_{\text {eff }}$ and $\mu_{\text {eff }}$ of the considered ZPC using effective medium theory, ${ }^{16}$ evidently highlights this fact that the Dirac point at $11.7 \mathrm{GHz}$ with $\mathbf{k}=0$ is the frequency at which the system effectively functions as a zero-index metamaterial $\left(\varepsilon_{\text {eff }}=\mu_{\text {eff }}=n_{\text {eff }}=0\right)$. In order to investigate the effect of the presence of ZPC on the transmission of the guided modes of the PC WG, the geometrical parameters of the PC WG are chosen in a way that the Dirac point frequency of the ZPC is placed almost at the center of the bandgap of the typical PC. Fig. 1(d) illustrates the projected band structure of the PC WG obtained by the plane-wave expansion method. ${ }^{17}$ From this figure, it is clear that the photonic bandgap of the PC WG extends from 10 to $15.3 \mathrm{GHz}$ at the $\Gamma$ point and the structure supports guided modes within 10.2 to almost $14 \mathrm{GHz}$ inside the bandgap. It is noteworthy that in order to achieve an efficient coupling between the modes around the
Dirac point frequency and the guided modes supported by the PC WG, the radius of the rods in the line defect of the PC WG is chosen as $r_{Z P C}$. In this case, the waveguide cannot be considered as single mode, ${ }^{25}$ and this choice gives rise to a pair of guided modes with different symmetries that are able to be efficiently coupled to the Dirac cone modes. Using the finite-difference time-domain method (FDTD), ${ }^{26}$ we calculated the transmission spectrum of light passing through the PC WG within $11 \mathrm{GHz}$ to $14 \mathrm{GHz}$. This range of frequencies falls inside the guiding band of the PC WG and, as expected, light achieves low resonant transmissions in this range due to support of the Fabry-Perot resonances ${ }^{3}$ (see weak peaks in the black-dotted curve in panel (a) of Fig. 2). In order to investigate the effect of the ZPC presence on the transmission of the $\mathrm{PC} \mathrm{WG}$, considering a typical value for $\Delta$ as $1.4 a_{P C}$, we add ZPC at the inner side of the waveguide. The dashed-red curve in Fig. 2(a) illustrates the transmission of this case. By comparing dotted and dashed curves in this figure, it is clearly seen that the presence of the ZPC at the inner side of the waveguide leads to almost 5 times of enhancement in transmission of the PC WG at $12.06 \mathrm{GHz}$, which is near the Dirac cone frequency of the ZPC. In fact, this enhancement happens because of strong couplings between the squeezed light into the ZPC at frequencies around the Dirac cone frequency and the FP resonances of the guided modes supported by the PC WG. Moreover, since the ZPC does not comprise any lossy inclusion, when we consider it at the inner and outer sides of the PC WG in the calculations, transmission of light through the system is enhanced by a factor of 9 at $12.12 \mathrm{GHz}$, as shown in the solid-blue curve in Fig. 2(a). This enhancement factor is 1.8 times larger than that obtained by using a grating-like corrugation at the inner side of the PC WG. ${ }^{23}$ It is worth noting that by taking $\Delta=a_{P C}$ as an optimal value and $L=12 a_{P C}$ where the 

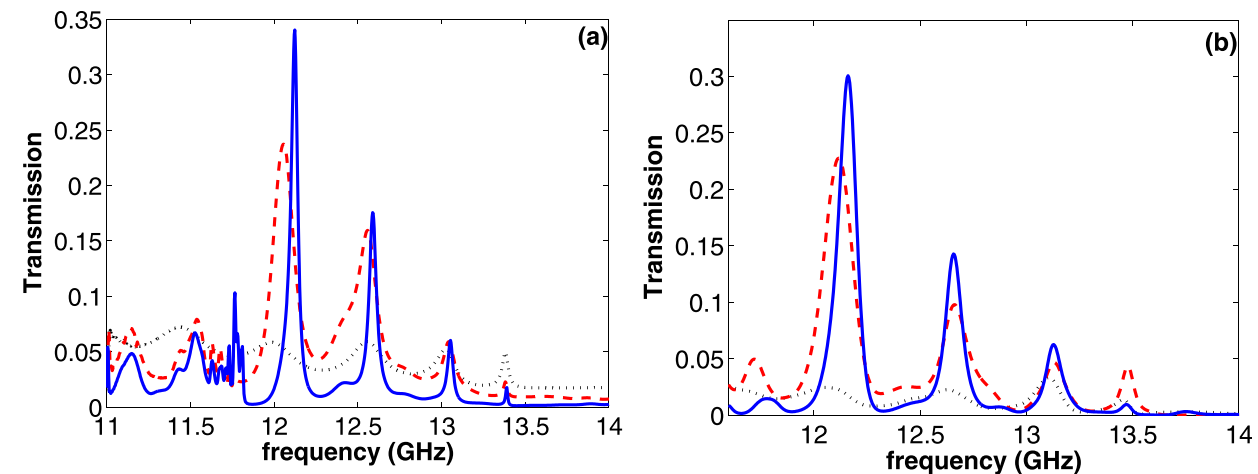

FIG. 2. Calculated (panel (a)) and measured (panel (b)) transmission spectrum of the PC WG (dotted), ZPC/PC WG (dashed-red), and ZPC/PC WG/ZPC (solid-blue) systems for a frequency region within the bandgap of the PC.

latter is the waveguide length, it is possible to increase the enhancement values of the resonant modes in Fig. 2 up to $50 \%$. Moreover, by increasing the waveguide length to this value, the number of resonant modes in this figure will be increased from 4 to 6 within 12 to $14 \mathrm{GHz}$. Notice that we chose $L_{\text {min }}$ as $8 a_{P C}$ in the presented results to be able to consider the structure as a PC WG. This enhancement mechanism is very similar to the one discussed in Ref. 3. However, in this letter, we not only experimentally investigate a feasible hybrid system, but also prove that the system under our consideration shows strong beaming, as illustrated in Figs. 3 and 4. It is noteworthy that at $f=12.12 \mathrm{GHz}, \varepsilon_{\text {eff }}=0.07$, $\mu_{\text {eff }}=0.17$ and thus $n_{\text {eff }}=\sqrt{\varepsilon_{\text {eff }} \mu_{\text {eff }}}=0.1091$ and $Z_{\text {eff }}=\mu_{\text {eff }}$ ' $\varepsilon_{\text {eff }}=1.5584$, which $Z_{\text {eff }}$ denotes the effective relative impedance of the ZPC. It is clear that, at this frequency, there is a small impedance mismatch between ZPC and free space. The value of the effective index of refraction at this frequency clearly verifies that the enhancement happens at an indexnear-zero (INZ) frequency. Similar to the first peak at 12.12 GHz of the solid-blue curve in Fig. 2(a), the second (a)

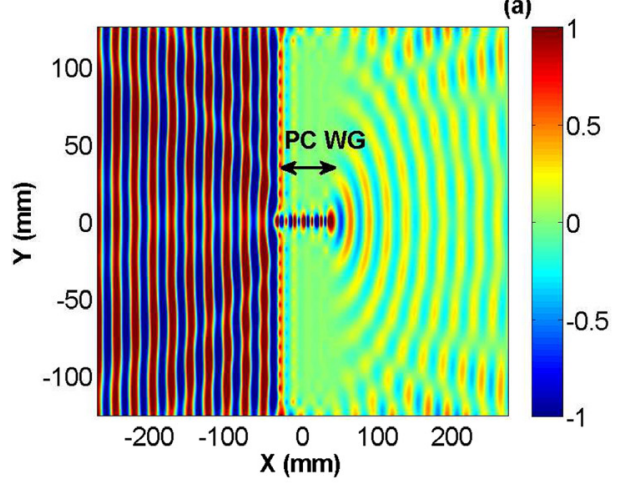

(b)

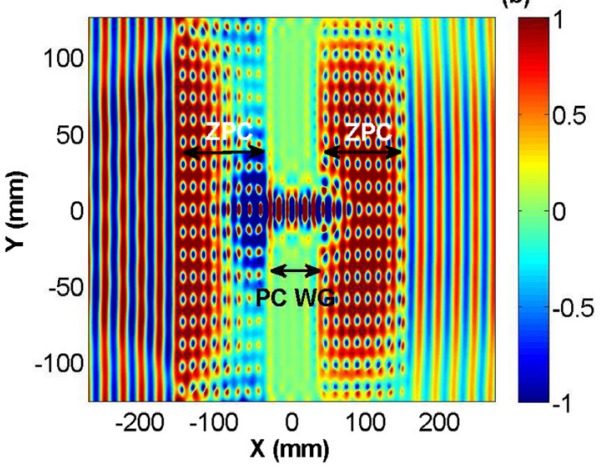

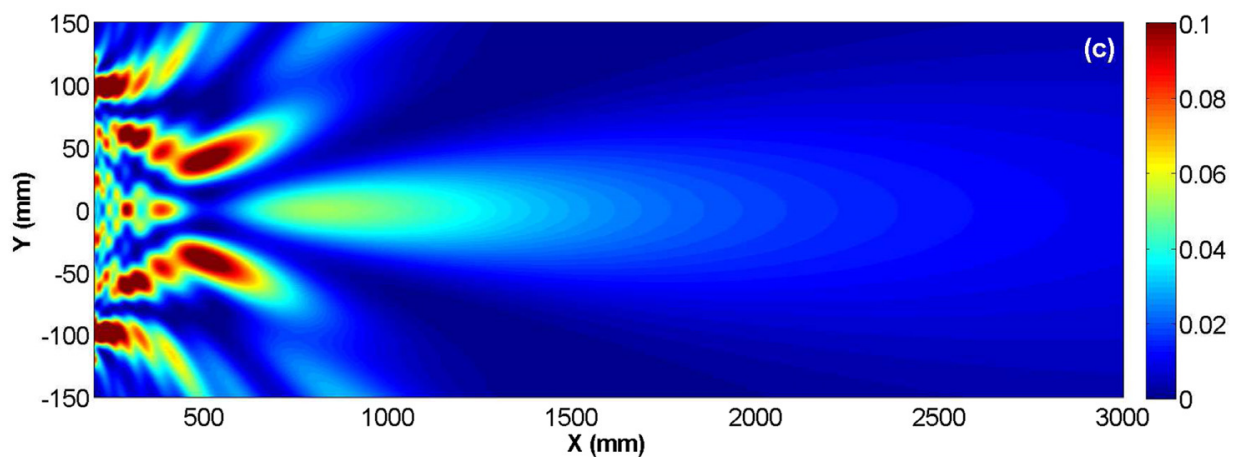

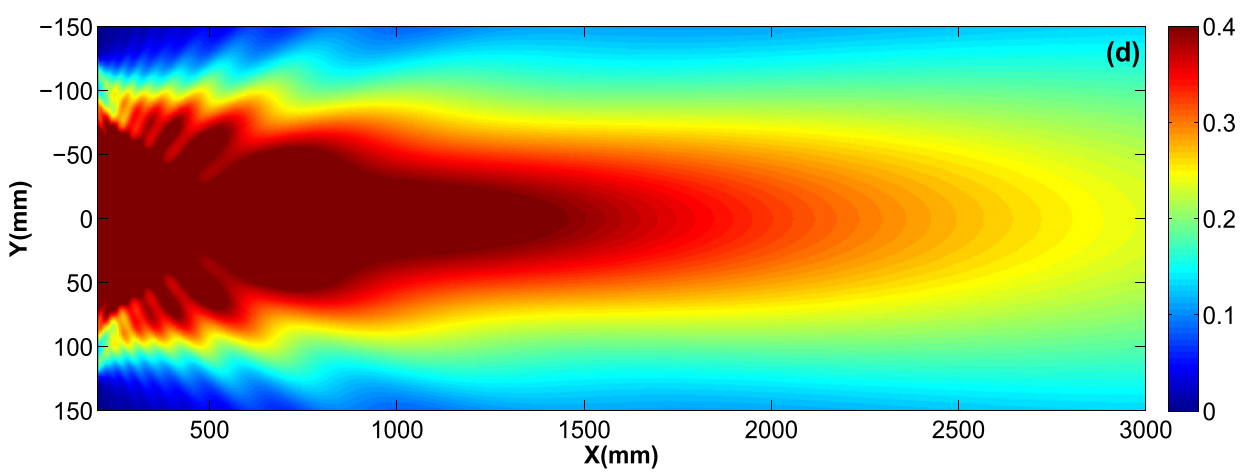

FIG. 3. Panels (a) and (b) show numerically simulated real part of $E_{z}$ component of the field distribution of a mode, at $\mathrm{f}=12.12 \mathrm{GHz}$, inside and outside of PC WG and hybrid ZPC/PC WG/ZPC systems, respectively. Panels (c) and (d), respectively, represent near-to-farfield transformations of the electric field intensity at $\mathrm{f}=12.12 \mathrm{GHz}$, for $\mathrm{PC}$ WG and ZPC/PC WG/ZPC structures. 

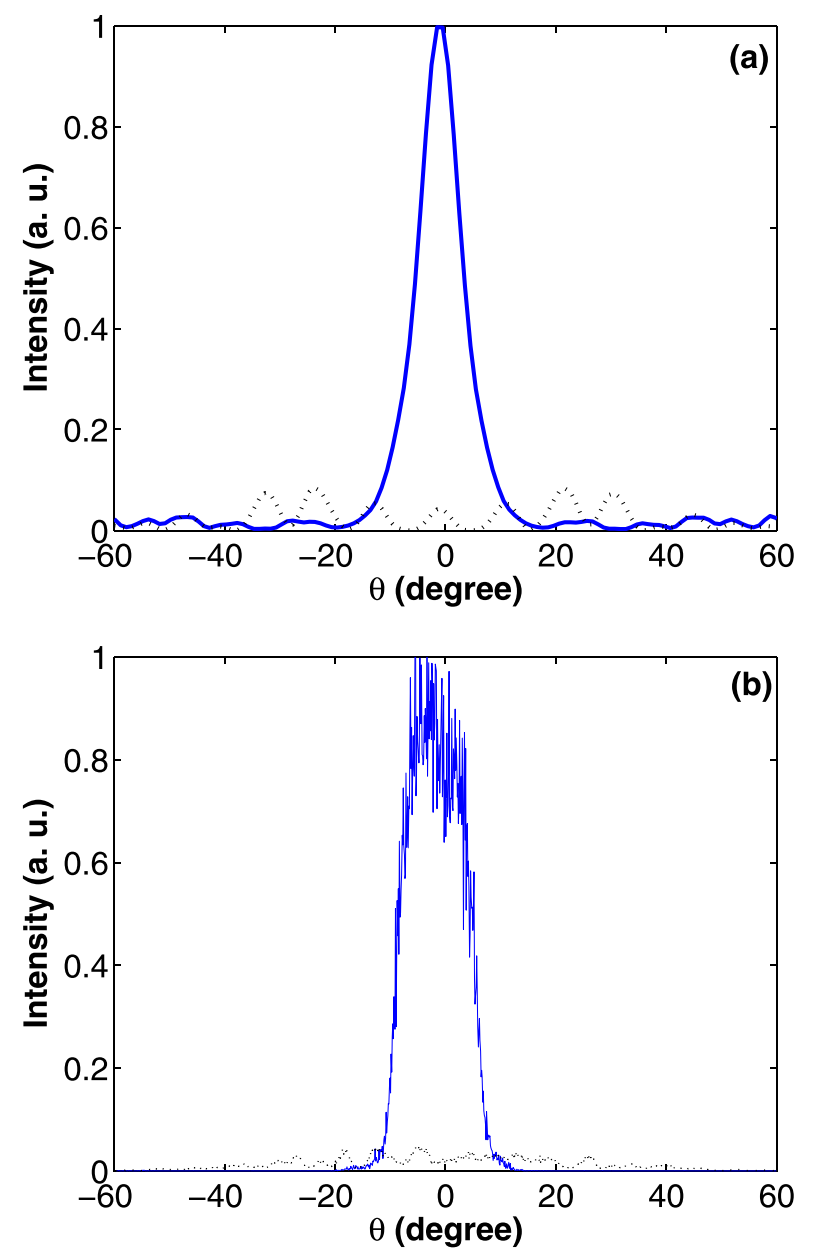

FIG. 4. Panels (a) and (b), respectively, illustrate calculated and measured far-field angular radiation pattern of the extracted light from the system at the guided-INZ mode, $\mathrm{f}=12.12 \mathrm{GHz}$.

weaker enhancement at $12.6 \mathrm{GHz}\left(n_{\text {eff }}=0.22, Z_{\text {eff }}=1.57\right)$ is also in connection with the very negligible impedance mismatching of the ZPC with free space and, consequently, the amplification of the amplitude of the electromagnetic field inside the ZPC at the INZ region. This amplification, in fact, leads to an excellent coupling between the incident light and the guided modes supported by the PC WG which finally exhibits itself as the considerable enhancements in the transmission spectrum. For this reason, we name these peaks in transmission as guided-INZ modes. In addition, in order to prove our theoretical results, using an experimental set up the same as that of explained in Refs. 23 and 24, we measured the transmission spectrum of the "PC WG," "ZPC/PC WG," and "ZPC/PC WG/ZPC" hybrid systems. The results are presented in Fig. 2(b) in dotted, dashed-red, and solidblue curves, respectively. As it is clear from this figure, the results obtained by experiments represent a perfect agreement with the numerical plots. Another critical point that also needs to be pointed out is that the width of the considered PC WG $(14.9 \mathrm{~mm})$ is smaller than the operating wavelength (around $25 \mathrm{~mm}$ ). As illustrated in Fig. 3(a), this feature causes a typical guided mode to be extracted from the "PC WG" with noticeable diffraction in all directions, and thus exhibits a very diffracted picture in far-field, see panel (c). Fig. 3(c) shows near-to-far-field (NTFF) transformation of a guided mode extracted from $\mathrm{PC} W G$ at $f=12.12 \mathrm{GHz}$. By adding surface corrugation and gratinglike layers to the outer side of the PC WG, the emitted light can be confined to a very narrow spatial region, called as highly directional emission or beaming. ${ }^{22-24}$ As it is evident from panel (b) of Fig. 3, having the zero-index photonic crystal at both sides of the PC WG, i.e., "ZPC/PC WG/ZPC" system, causes an incident plane wave on the structure, at a guide-INZ frequency, passes through the system with no change in its spatial phase. This is the direct consequence of a very small value of the wave vector, $|\mathbf{k}| \ll \mathbf{k}_{0}$, inside the ZPC for a guided-INZ mode; at $f=12.12 \mathrm{GHz},|\mathbf{k}|$ $=0.1091 \mathbf{k}_{0}$ inside the ZPC. Consequently, the presence of the ZPC at both sides of the PC WG exhibits not only the significant enhancements in the light transmission through the PC waveguide but it can also directionally beam the extraction of electromagnetic waves at far-field, as presented in Fig. 3(d). As it is obvious from this panel, the electric field intensity is confined to a very narrow spatial region and consequently shows significant beaming. This is the most distinguished characteristic of a ZPC. In other words, the extracted light from the hybrid system propagates without diffracting into a wide angular region and thus, as shown in Fig. 4, a strong directionality in the far-field radiation pattern. Figure 4(a) shows the calculated far-field radial distribution of the electric field intensity of the PC WG without (dotted-black curve) and with the presence of the ZPC at both sides of it (solid-blue curve), at $f=12.12 \mathrm{GHz}$. In support of these results, the measured data of the mentioned systems are represented in panel (b). It should be noted that, in our experimental setup, the receiver antenna is connected to a rotating arm in order to measure the angular dependence of the far-field radiation. Comparing Figs. 4(a) and 4(b), it can be observed that (i) there is a good agreement between the results obtained by simulation and experiment. (ii) With the presence of the zero-index photonic crystal at the outer side of the waveguide, the emitted power is confined to a very narrow angular region with a half-power width of $14^{\circ}$ (from experiment) compared with the widespread emitted power of the typical PC WG. Consequently, the presence of the ZPC at both sides of the $\mathrm{PC} \mathrm{WG}$, in addition to considerably enhancing light transmission through the waveguide, noticeably beams its extraction from the hybrid system at INZ frequencies.

In conclusion, we have experimentally demonstrated that using an all-dielectric zero-index photonic crystal at the inner side of a typical PC waveguide can lead to a considerable enhancement in the transmission spectrum of the hybrid ZPC/PC WG system. We proved that this enhancement happens at frequencies near the Dirac frequency of the ZPC, at which the impedance mismatching between the ZPC and free space is pretty small. Moreover, it has been shown that the presence of the ZPC at both sides of the PC WG, in addition to enhancing the transmission, leads to the strong directional beaming of light extracted from this system. This suggested hybrid structure can be potentially useful as a basic component in devices that are beneficial for near-field microscopy, lasing, and light extraction from LEDs.

This work was supported by Project Nos. TUBITAK114E505, DPT-HAMIT, NATO-SET-193, TUBITAK-113E331, 
and TUBITAK-114E374. The authors (E.O. and H.C.) also acknowledge the partial support from the Turkish Academy of Sciences.

${ }^{1}$ R. W. Ziolkowski, Phys. Rev. E 70, 046608 (2004).

${ }^{2}$ M. Silveirinha and N. Engheta, Phys. Rev. Lett. 97, 157403 (2006).

${ }^{3}$ A. Chen, Y. Fu, Y. Xu, and H. Chen, J. Opt. 17, 105602 (2015).

${ }^{4}$ M. Silveirinha and N. Engheta, Phys. Rev. B 75, 075119 (2007).

${ }^{5}$ A. Alu, M. G. Silveirinha, A. Salandrino, and N. Engheta, Phys. Rev. B 75, 155410 (2007).

${ }^{6}$ Y. Fu, L. Xu, Z. Hong Hang, and H. Chen, Appl. Phys. Lett. 104, 193509 (2014).

${ }^{7}$ Y. Fu, Y. Xu, and H. Chen, Sci. Rep. 5, 11217 (2015).

${ }^{8}$ J. Hao, W. Yan, and M. Qiu, Appl. Phys. Lett. 96, 101109 (2010).

${ }^{9}$ Y. Xu and H. Chen, Appl. Phys. Lett. 98, 113501 (2011).

${ }^{10}$ A. Alu, F. Bilotti, N. Engheta, and L. Vegni, IEEE Trans. Antennas Propag. 54, 1632 (2006).

${ }^{11}$ J. B. Pendry, Science 306, 1353-1355 (2004).

${ }^{12}$ R. Liu, Q. Cheng, T. Hand, J. J. Mock, T. J. Cui, S. A. Cummer, and D. R. Smith, Phys. Rev. Lett. 100, 023903 (2008).

${ }^{13}$ X. Huang, Y. Lai, Z. H. Hang, H. Zheng, and C. T. Chan, Nat. Mater. 10, $582(2011)$.
${ }^{14}$ K. S. Novoselov, A. K. Geim, S. V. Morozov, D. Jiang, M. I. Katsnelson, I. V. Grigorieva, S. V. Dubonos, and A. A. Firsov, Nature 438, 197-200 (2005).

${ }^{15}$ X. Zhang, Phys. Rev. Lett. 100, 113903 (2008).

${ }^{16}$ Y. Wu, J. Li, Z. Q. Zhang, and C. T. Chan, Phys. Rev. B 74, 085111 (2006).

${ }^{17}$ J. D. Joannopoulos, S. G. Johnson, J. N. Winn, and R. D. Meade, Photonic Crystals: Molding the Flow of Light, 2nd ed. (Princeton University Press, Princeton, NJ, 2008).

${ }^{18}$ F. Dominec, C. Kadlec, H. Nemec, P. Kuzel, and F. Kadlec, Opt. Express 22, 30492 (2014).

${ }^{19}$ M. V. Rybin, D. S. Filonov, K. B. Samusev, P. A. Belov, Y. S. Kivshar, and M. F. Limonov, Nat. Commun. 6, 10102 (2015).

${ }^{20}$ P. Moitra, Y. Yang, Z. Anderson, I. I. Kravchenko, D. P. Briggs, and J. Valentine, Nat. Photonics 7, 791 (2013).

${ }^{21}$ Y. Li, S. Kita, P. Muñoz, O. Reshef, D. I. Vulis, M. Yin, M. Loncar, and E. Mazur, Nat. Photonics 9, 738 (2015).

${ }^{22}$ E. Moreno, F. J. Garcia-Vidal, and L. Martin-Moreno, Phys. Rev. B 69, 121402(R) (2004).

${ }^{23}$ I. Bulu, H. Caglayan, and E. Ozbay, Opt. Lett. 30, 3078 (2005).

${ }^{24}$ H. Caglayan, I. Bulu, and E. Ozbay, Appl. Phys. Lett. 92, 092114 (2008).

${ }^{25}$ S. G. Johnson, P. R. Villeneuve, S. Fan, and J. D. Joannopoulos, Phys. Rev. B 62, 8212 (2000).

${ }^{26}$ See http://www.lumerical.com/tcad-products/fdtd/ for Lumerical Solutions, Inc. 